\title{
Efeitos metabólicos da glutamina em ratos submetidos à queimadura por água fervente (escaldadura) ${ }^{1}$
}

\author{
Rômulo Cesar Costa Barbosa ${ }^{2}$ \\ Sergio Botelho Guimarães ${ }^{3}$ \\ Paulo Roberto Cavalcante de Vasconcelos ${ }^{4}$ \\ Carolyne Rolim Chaves ${ }^{4}$ \\ Paulo Roberto Leitão de Vasconcelos ${ }^{5}$
}

\begin{abstract}
Barbosa RCC, Guimarães SB, Vasconcelos PRC, Chaves CR, Vasconcelos PRL. Efeitos metabólicos da glutamina em ratos submetidos à queimadura por água fervente (escaldadura). Acta Cir Bras [serial online] 2003 Nov-Dez;18(6). Disponível em URL: http://www.scielo.br/acb.

RESUMO - Objetivo: Investigar os efeitos metabólicos da L-glutamina (Gln) em ratos anestesiados submetidos à queimadura por água fervente. Métodos: Foram estudados vinte e quatro ratos Wistar machos, anestesiados, submetidos a queimaduras da pele dorsal após exposição à água quente $\left(100^{\circ} \mathrm{C}\right)$ durante 10 segundos $(30 \%$ de superfície corporal). Os ratos foram randomizados para receber, por gavagem, $2 \mathrm{ml}$ de água (G-1) ou igual volume de solução de Gln (0,5g/peso/dia) (G-2). Amostras de tecido (pele sadia e queimada, músculo e fígado) e sangue foram coletadas 24h (D1) e 48h (D2) pós-trauma para análise enzimática. Resultados: A oferta de Gln induziu aumento significante nas concentrações de glicose na pele saudável em animais do G-2 no D2, e na pele queimada em G-2/D1. As concentrações de lactato também aumentaram significantemente em G-2/D1 no músculo $(11,29 \pm 1,25 \mu \mathrm{mol} / \mathrm{g}$ contra 7,43 $\pm 0,93 \mu \mathrm{mol} / \mathrm{g}-\mathrm{p}<0,05)$ e no G-2/D2 no fígado $(7,68 \pm 1,49 \mu \mathrm{mol} / \mathrm{g}$ contra $3,27 \pm 0,67 \mu \mathrm{mol} / \mathrm{g} \mathrm{p}<0,01)$, e em pele sadia $(5,30 \pm 0,42 \mu \mathrm{mol} / \mathrm{g}$ contra $3,57 \pm 0,38 \mu \mathrm{mol} / \mathrm{g}-\mathrm{p}<0,05)$. As concentrações de piruvato diminuíram significantemente no grupo G-2/D1 (músculo e fígado) e aumentaram na pele sadia no grupo G-2/D2. As concentrações de ATP diminuíram significantemente no músculo em G-2 nos tempos D1 e D2. Conclusões: A oferta de Gln sinaliza para utilização de piruvato para glicólise preponderantemente anaeróbica no músculo. $\mathrm{O}$ aumento nas concentrações de lactato tecidual pode vir a ser decorrente da oferta exógena de Gln, que transformada em glutamato, leva à ativação do ciclo malato-aspartato com conseqüente favorecimento da glicólise anaeróbica. A oferta de Gln induz a possível aumento na captação de glicose tanto por pele sadia quando na pele queimada. Houve falha de desenvolvimento da hipercetonemia adaptativa ao jejum em ambos os grupos. A oferta exógena de Gln parece não induzir alteração na ureagênese.
\end{abstract}

DESCRITORES - Queimaduras. Metabolismo. Glutamina. Ratos.

\section{Introdução}

Queimaduras são lesões térmicas resultantes da transferência de energia de uma fonte de calor para o corpo.

Os principais fatores que determinam a sobrevivência após queimaduras são: a porcentagem da superfície queimada, a profundidade das lesões, a localização, o tipo do agente causal, lesões por inalação, a idade do paciente, o estado nutricional e doenças metabólicas pré-existentes. ${ }^{1}$

A glutamina desempenha papel fundamental no metabolismo dos aminoácidos. Em razão de sua biaminação atua como transportadora de nitrogênio e no transporte intracelular do ácido glutâmico, pois a membrana celular é mais permeável a glutamina que ao ácido glutâmico, sendo precursora de vários aminoácidos. ${ }^{2}$ Tra- balhos realizados nas décadas de 50 e 60 tornaram claro que a função metabólica da glutamina não se limita a um precursor da síntese protéica, mas que funciona como um importante mediador numa variedade de vias metabólicas em diversos tipos celulares. ${ }^{3}$

Diante da magnitude das repercussões metabólicas das queimaduras objetivou-se estudar os efeitos da L-glutamina

1. Trabalho realizado no Laboratório de Cirurgia Experimental (LABCEX), Programa de Pós-Graduação Stricto Sensu em Cirurgia, Faculdade de Medicina, Departamento de Cirurgia da Universidade Federal do Ceará (UFC).

2. Cirurgião Plástico, Mestrando em Cirurgia Experimental do Departamento de Cirurgia, UFC.

3. Mestre em Cirurgia, Doutorando em Cirurgia Experimental e Professor Adjunto do Departamento de Cirurgia, UFC.

4. Estudantes de Medicina da UFC.

5. Professor Doutor $(P h D)$, Coordenador do Curso de Pós-Graduação Stricto Sensu em Cirurgia da Faculdade de Medicina da UFC. 
administrada, por gavagem, sobre as concentrações in vivo sangüíneas, cutâneas, musculares e hepáticas dos metabólitos glicose, piruvato, lactato, corpos cetônicos (acetoacetato + 3-hidroxibutirato) e ATP, e as concentrações de uréia urinária de $24 \mathrm{~h}$, em ratos submetidos à queimadura por água fervente.

\section{Métodos}

A pesquisa experimental foi realizada de acordo com os preceitos do Colégio Brasileiro de Experimentação Animal (COBEA) e as normas do CIOMS (Council for International Organization of Medical Sciences) ethical code for animal experimentation (WHO Chronicle 1985; 39(2):51-6).

Foram utilizados vinte e quatro (24) ratos (Mammalia Rodentia, Muridae, Rattus Norvegicus Albinus), da linhagem Wistar, machos, adultos, saudáveis e bem nutridos, com peso médio de $390 \mathrm{~g}$ ( $320 \mathrm{~g}$ a $480 \mathrm{~g}$ ), procedentes do Biotério Central da Universidade Federal do Ceará. Os 24 animais foram distribuídos em dois grupos $(\mathrm{n}=12)$, classificados como: Grupo 1 controle - água; e Grupo 2 experimento L-glutamina (Gln). Os animais foram mantidos no Biotério do Laboratório de Cirurgia Experimental (LABCEX) do Departamento de Cirurgia da Faculdade de Medicina da Universidade Federal do Ceará, alojados em ambiente com ar refrigerado, à temperatura média de $25^{\circ} \mathrm{C}$, umidade relativa do ar em torno de $50 \%$, iluminação adequada de acordo com o ciclo circadiano, e alimentados com ração balanceada tipo Purina e água ad libitum, até antes do início do experimento. O experimento foi desenvolvido nas seguintes etapas: 1) queimadura de $30 \%$ da área corpórea; 2) jejum com oferta de água $a b$ libitum, e administração de L-glutamina ou água, por gavagem, $18 \mathrm{~h}$ e $42 \mathrm{~h}$ após o trauma térmico, e colheita de urina de 24 horas; 3) procedimento cirúrgico para colheita das amostras (pele queimada do dorso, pele sadia do abdome, musculatura do membro traseiro esquerdo e lobo esquerdo do fígado) realizada em dois tempos distintos (24h e $48 \mathrm{~h}$ após o trauma térmico); 4) dosagens dos metabólitos nas amostras teciduais e de sangue arterial (ATP, glicose, piruvato, lactato e corpos cetônicos) e dosagem de uréia urinária; 5) análise estatística dos resultados das concentrações in vivo das substâncias dosadas. Após colheita, com o animal ainda anestesiado, ocorria a eutanásia do animal por punção aspirativa exsanguinante da aorta abdominal.

\section{Obtenção da queimadura}

Depois de anestesiado (anestesia inalatória com éter dietílico), o animal era colocado em decúbito dorsal sobre um molde único de borracha isolante medindo $25 \times 15 \times 5 \mathrm{~cm}$, com abertura central (baseado em modificação do modelo publicado por Walker e col.,1968). ${ }^{4}$ O tamanho dos animais utilizados no estudo permitia apenas a exposição de parte delimitada do dorso correspondente a uma área de $30 \%$ da superfície corporal total. Em seguida, o molde e o animal eram imersos por um período de dez segundos em caixa metálica das mesmas dimensões, posicionada sobre aquecedor com termostato, contendo água a uma temperatura constante de $100^{\circ} \mathrm{C}$, sem o animal apresentar sinais de dor ou estresse, resultando numa queimadura de terceiro grau, não letal, de espessura total da pele do dorso, com conseqüente destruição das terminações cutâneas nervosas sensitivas, resultando uma lesão indolor. ${ }^{5} \mathrm{~A}$ superfície corporal queimada no dorso do animal foi padronizada com base no peso médio da amostra sendo a área da abertura central calculada em centímetros quadrados pela fórmula: 0,7854 x maior diâmetro x menor diâmetro, correspondendo a uma superfície corporal queimada aproximada de trinta por cento em relação à superfície corporal total do animal, calculada em centímetros quadrados pela fórmula: $\left(11 \mathrm{x}\right.$ peso $\left.{ }^{0,631}\right) .^{6,7}$

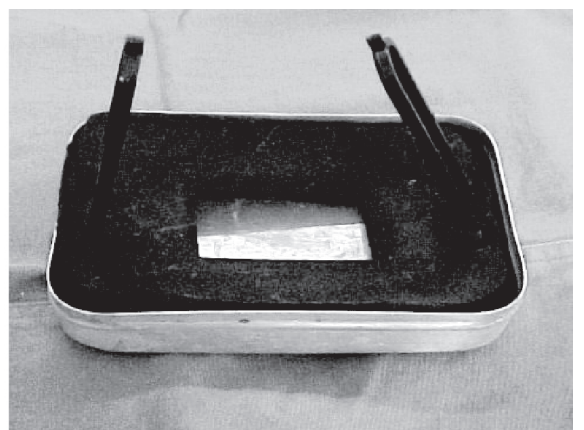

FIGURA 1 - Modelo de molde borracha isolante usado para isolar a área a ser queimada, no rato (segundo Walker e col., 1968 modificado). ${ }^{4}$
Este modelo utiliza materiais simples e de baixo custo, sendo facilmente reproduzido, desde que sejam utilizados animais de pesos semelhantes aos usados neste estudo, previamente anestesiados, proporcionando uma queimadura uniforme, reprodutível e indolor, no período póslesão térmica (Figura 1).

Após o procedimento foi realizada reidratação imediata por injeção subcutânea de solução salina a $0,9 \%$ na dose de $1 \mathrm{ml} / \mathrm{Kg}$ peso/superfície corporal queimada (S.C.Q.). A anestesia foi mantida, durante todo o procedimento, por técnica de máscara aberta com introdução intermitente das narinas do animal em tubo de plástico, de tamanho compatível, contendo chumaço de algodão embebido em éter dietílico

Após a lesão térmica, os animais foram alojados em gaiolas metabólicas individuais de aço para coleta da urina de 24 horas, e mantidos em jejum, sem restrição hídrica, no Biotério do LABCEX, nas mesmas condições anteriormente descritas, obedecendo a seguinte distribuição:

\section{Grupo 1 - água}

(Dia 1) - 6 animais receberam por gavagem $2 \mathrm{ml}$ de água 18 horas após o trauma e foram sacrificados ao completarem 24 horas da queimadura, com colheita da urina de 24 horas.

(Dia 2) - 6 animais receberam por gavagem $2 \mathrm{ml}$ de água 18 horas e 42 horas após o trauma e foram sacrificados ao completarem 48 horas da queimadura, com colheita da urina a cada 24 horas.

\section{Grupo 2 - L-glutamina (Gln)}

(Dia 1) - 6 animais receberam por gavagem uma solução de L-glutamina (0,5 $\mathrm{g} / \mathrm{Kg}$ peso/dia) diluída em $2 \mathrm{ml}$ de água 18 horas após o trauma e foram sacrificados ao completarem 24 horas da queimadura, com colheita da urina de 24 horas.

(Dia 2) - 6 animais receberam por gavagem uma solução de L-glutamina $(0,5$ $\mathrm{g} / \mathrm{Kg}$ peso/dia) diluída em $2 \mathrm{ml}$ de água 18 horas e 42 horas após o trauma e foram sacrificados ao completarem 48 horas da queimadura, com colheita da urina a cada 24 horas.

Foi utilizada L-glutamina na forma de pó, diluída em $2 \mathrm{ml}$ de água, fabricada pelo laboratório Ajinomoto. A urina foi colhida sem conservantes, em frascos individuais 
posicionados abaixo de cada gaiola metabólica.

Os metabólitos no sangue da aorta e nos tecidos (fígado, músculo e pele) foram determinados por métodos de ensaio enzimático. ${ }^{8,9,10}$ Foram incluídos neste estudo o trifosfato de adenosina (ATP), os produtos da glicólise (piruvato e lactato), os corpos cetônicos (acetoacetato e 3-hidroxibutirato) e a D-glicose. As concentrações dos metabólitos foram calculadas em $\mu \mathrm{mol} / \mathrm{g}$ tecido fresco ou $\mu \mathrm{mol} / \mathrm{ml}$ de sangue. A urina colhida individualmente a cada período de 24 horas foi encaminhada imediatamente ao Laboratório Central do Hospital Universitário Walter Cantídio da Universidade Federal do Ceará, para determinação da uréia por método enzimático (UVurease). ${ }^{11}$ Os resultados foram expressos como média \pm E.P.M. (Erro Padrão da Média), acompanhados pelo número de observações (n).

A significância estatística foi calculada conforme o teste não paramétrico Mann-Whitney. O nível de significância estabelecido foi de $\mathrm{p}<0,05$.

\section{Resultados}

Não ocorreram alterações significantes nas concentrações hepáticas e cutâneas (pele sadia e pele queimada) de ATP em nenhum dos grupos estudados. No tecido muscular ocorreu redução significante nas concentrações de ATP nos animais tratados com Gln no G-2, nos dias 1 e 2 pós-queimadura, quando comparadas às aferidas no grupo G-1 (Tabela 1).

Não ocorreram alterações significantes nas concentrações de glicose no fígado, músculo e sangue em nenhum dos grupos estudados. Entretanto, houve aumento significante nas concentrações de glicose na pele sadia dos ratos tratados com Gln, no dia 2 pós-queimadura em comparação com o grupo controle. $\mathrm{Na}$ pele queimada houve aumento significante nas concentrações de glicose no grupo tratado com Gln em relação ao controle no dia 1 pós-queimadura. Houve uma diminuição nas concentrações deste metabólito no dia 2 quando comparadas às aferidas no dia 1 no mesmo grupo 2 (Tabela 2).

No presente estudo não foram encontradas alterações significantes nas concentrações de piruvato no sangue em nenhum dos grupos estudados. Houve, no entanto, uma queda nas concentrações de piruvato no fígado no dia 1 no grupo que utilizou Gln em comparação com as concentrações encontradas no grupo controle (Tabela 3). No dia 1 pós-trauma térmico houve uma queda nas concentrações de piruvato no músculo no grupo tratado com Gln em relação às obtidas no grupo controle (Tabela 3). Ocorreu aumento significante das concentrações de piruvato na pele sadia dos ratos tratados com Gln, no dia 2, em relação ao dia 1 . No dia 2, houve também elevação significante no grupo tratado com Gln em relação ao respectivo controle (G-1). (Tabela 3). Houve redução significante nas concentrações de piruvato na pele queimada dos animais do grupo controle G-1 no dia 2 em relação ao dia 1 (Tabela 3 ).

Não foram observadas alterações na lactacemia em nenhum dos grupos G-1 e G-2 em nenhum dos tempos estudados (G1 em D1 - 4,990 $\pm 1,030 \mu \mathrm{mol} / \mathrm{ml}$ versus $\mathrm{G}-2$ em D1 -5,506 $\pm 0,387 \mu \mathrm{mol} / \mathrm{ml}$; G-1 em D2 $-3,218 \pm 0,979 \mu \mathrm{mol} / \mathrm{ml}$ versus $\mathrm{G}-2 \mathrm{em} \mathrm{D} 2$ $-4,810 \pm 1,320 \mu \mathrm{mol} / \mathrm{ml})$. Houve aumento das concentrações de lactato hepático do grupo Gln (G-2) no dia 2 em comparação ao G-1 (Figura 4). Houve aumento significante nas concentrações de lactato muscular nos animais tratados com Gln, em comparação às concentrações aferidas no G-1, no dia 2 de pós-queimadura (Figura 2).

Houve aumento significante nas concentrações de lactato na pele sadia dos ratos tratados com Gln (G-2), no dia 1, em comparação às concentrações aferidas no G-1, no mesmo tempo (Figura 3).

TABELA 1 - Concentrações de ATP ( $\mu \mathrm{mol} / \mathrm{mg}$ de tecido fresco) no fígado, pele sadia, pele queimada e músculo

\begin{tabular}{ccccccccc}
\hline & \multicolumn{2}{c}{ Fígado } & \multicolumn{2}{c}{ Pele sadia } & \multicolumn{2}{c}{ Pele queimada } & \multicolumn{2}{c}{ Músculo } \\
& $\mathbf{1}$ & $\mathbf{2}$ & $\mathbf{1}$ & $\mathbf{2}$ & $\mathbf{1}$ & $\mathbf{2}$ & $\mathbf{1}$ & $\mathbf{2}$ \\
& Dia & Dia & Dia & Dia & Dia & Dia & Dia & Dia \\
\hline Grupo 1 & 0,005 & 0,004 & 0,008 & 0,008 & 0,009 & 0,0055 & 0,010 & 0,009 \\
(Água) & \pm & \pm & \pm & \pm & \pm & \pm & \pm & \pm \\
& 0,001 & 0,001 & 0,001 & 0,002 & 0,001 & 0,001 & 0,002 & 0,001 \\
& & & & & & & \\
Grupo 2 & 0,005 & 0,007 & 0,003 & 0,004 & 0,012 & 0,007 & $0,004^{*}$ & $0,002^{*}$ \\
(Gln) & \pm & \pm & \pm & \pm & \pm & \pm & \pm & \pm \\
& 0,001 & 0,003 & 0,002 & 0,001 & 0,002 & 0,004 & 0,001 & 0,001 \\
\hline
\end{tabular}

$* \mathrm{p}<0,05$ quando comparado ao respectivo controle (G-1).

Resultados expressos como Média \pm E.P.M. ( $\mathrm{n}=6$, cada subgrupo)
Observou-se redução significante das concentrações de lactato em pele sã no dia 2 , em relação às concentrações aferidas no dia 1 , nos animais tratados com Gln (G-2) (Figura 3). Não houve alteração significante nas concentrações de lactato em pele queimada quando comparados os grupos G-1 e G-2 em nenhum dos tempos estudados.

Não foram observadas alterações nas concentrações de uréia urinária em nenhum dos grupos G-1 e G-2 em nenhum dos tempos pós-trauma térmico estudados (Figura 4).

Não foram observadas alterações na cetonemia, nem nas concentrações de corpos cetônicos no fígado, músculo, pele sadia e pele queimada em nenhum dos grupos estudados (Tabela 4).

\section{Discussão}

Dentre as várias formas de trauma, as grandes queimaduras se destacam como o maior estado hipercatabólico, constante e prolongado. ${ }^{12}$ As queimaduras determinam grandes alterações metabólicas resultantes da combinação da liberação de mediadores inflamatórios e resposta hormonal induzidas pelo estresse. A gliconeogênese é notadamente elevada após queimaduras. ${ }^{1} \mathrm{~A}$ oferta de glicose exógena apenas ocasiona uma redução em torno de 50\% na sua produção (gliconeogênese). As causas deste descontrole são um aumento na produção, uma perda da ação supressiva da glicose exógena na produção endógena deste substrato e uma queda da eficácia da insulina ou intolerância à glicose. ${ }^{12}$ Simultaneamente, ocorre uma grande mobilização de 
TABELA 2 - Concentrações de glicose ( $\mu \mathrm{mol} / \mathrm{mg}$ de tecido fresco) no fígado, pele sadia, pele queimada e sangue ( $\mu \mathrm{mol} / \mathrm{mg}$ de tecido fresco)

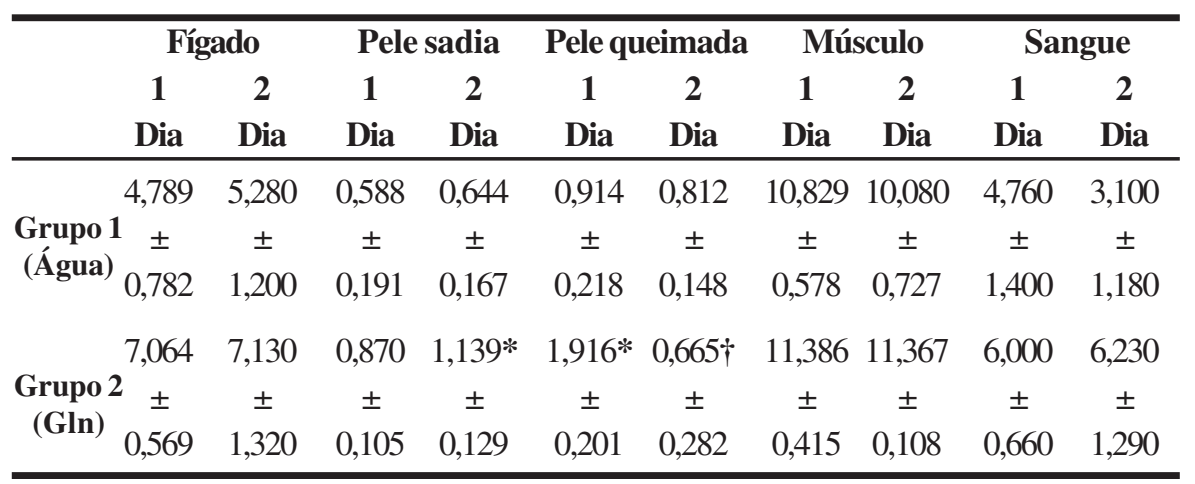

* $\mathrm{p}<0,05$ quando comparado ao respectivo controle (G-1)

$\dagger \mathrm{p}<0,05$ quando comparado ao Dia 1 (G-2)

Resultados expressos como Média \pm E.P.M. (n=6, cada subgrupo)

TABELA 3 - Concentração de piruvato ( $\mu \mathrm{mol} / \mathrm{mg}$ de tecido fresco) no fígado, pele sadia, pele queimada e sangue ( $\mu \mathrm{mol} / \mathrm{mg}$ de tecido fresco) fresco)

\begin{tabular}{|c|c|c|c|c|c|c|c|c|c|}
\hline \multicolumn{2}{|c|}{ Fígado } & \multicolumn{2}{|c|}{ Pele sadia } & \multicolumn{2}{|c|}{ Pele queimada } & \multicolumn{2}{|c|}{ Músculo } & \multicolumn{2}{|c|}{ Sangue } \\
\hline $\begin{array}{c}1 \\
\text { Dia }\end{array}$ & $\begin{array}{c}2 \\
\text { Dia }\end{array}$ & $\begin{array}{c}1 \\
\text { Dia }\end{array}$ & $\begin{array}{c}2 \\
\text { Dia }\end{array}$ & $\begin{array}{c}1 \\
\text { Dia }\end{array}$ & $\begin{array}{c}2 \\
\text { Dia }\end{array}$ & $\begin{array}{c}1 \\
\text { Dia }\end{array}$ & $\begin{array}{c}2 \\
\text { Dia }\end{array}$ & $\begin{array}{c}1 \\
\text { Dia }\end{array}$ & $\begin{array}{c}2 \\
\text { Dia }\end{array}$ \\
\hline $\begin{array}{l}0,173 \\
\text { rrupo } 1 \\
\text { (Água) } \\
0,016\end{array}$ & $\begin{array}{c}0,124 \\
\pm \\
0,038\end{array}$ & $\begin{array}{c}0,014 \\
\pm \\
0,002\end{array}$ & $\begin{array}{c}0,010 \\
\pm \\
0,004\end{array}$ & $\begin{array}{c}0,097 \\
\pm \\
0,067\end{array}$ & $\begin{array}{c}0,017 * \\
\pm \\
0,006\end{array}$ & $\begin{array}{c}0,040 \\
\pm \\
0,006\end{array}$ & $\begin{array}{c} \pm \\
0,008\end{array}$ & $\begin{array}{c}0,037 \\
\pm \\
0,009\end{array}$ & $\begin{array}{c} \pm \\
0,005\end{array}$ \\
\hline 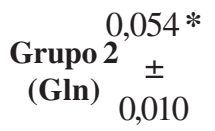 & $\begin{array}{c}0,051 \\
\pm \\
0,011\end{array}$ & $\begin{array}{c}0,006 \\
\pm \\
0,001\end{array}$ & $\begin{array}{c}0,022 \dagger \\
\pm \\
0,004\end{array}$ & $\begin{array}{c}0,036 \\
\pm \\
0,001\end{array}$ & $\begin{array}{c}0,019 \\
\pm \\
0,004\end{array}$ & $\begin{array}{c}0,008 * \\
\pm \\
0,003\end{array}$ & $\begin{array}{c}0,057 \\
\pm \\
0,041\end{array}$ & $\begin{array}{c}0,038 \\
\pm \\
0,004\end{array}$ & $\begin{array}{c}0,033 \\
\pm \\
0,007\end{array}$ \\
\hline
\end{tabular}

$* \mathrm{p}<0,05$ quando comparado ao respectivo controle (G-1)

$\dagger \mathrm{p}<0,05$ quando comparado ao Dia 1 (G-2)

Resultados expressos como Média \pm E.P.M. (n=6, cada subgrupo)

TABELA 4 - Concentrações de corpos cetônicos ( $\mu \mathrm{mol} / \mathrm{g}$ de tecido fresco) no fígado, músculo pele sadia, pele queimada e sangue $(\mu \mathrm{mol} / \mathrm{ml})$

\begin{tabular}{|c|c|c|c|c|c|c|c|c|c|c|}
\hline & \multicolumn{2}{|c|}{ Fígado } & \multicolumn{2}{|c|}{ Pele sadia } & \multicolumn{2}{|c|}{ Pele queimada } & \multicolumn{2}{|c|}{ Músculo } & \multicolumn{2}{|c|}{ Sangue } \\
\hline & $\begin{array}{c}1 \\
\text { Dia }\end{array}$ & $\begin{array}{c}2 \\
\text { Dia }\end{array}$ & $\begin{array}{c}1 \\
\text { Dia }\end{array}$ & $\begin{array}{c}2 \\
\text { Dia }\end{array}$ & $\begin{array}{c}1 \\
\text { Dia }\end{array}$ & $\begin{array}{c}2 \\
\text { Dia }\end{array}$ & $\begin{array}{c}1 \\
\text { Dia }\end{array}$ & $\begin{array}{c}2 \\
\text { Dia }\end{array}$ & $\begin{array}{c}1 \\
\text { Dia }\end{array}$ & $\begin{array}{c}2 \\
\text { Dia }\end{array}$ \\
\hline & $\begin{array}{c}0,309 \\
\mathbf{1} \\
\pm \\
0,008\end{array}$ & $\begin{array}{c} \pm \\
0,026\end{array}$ & $\begin{array}{c}0,068 \\
\pm \\
0,011\end{array}$ & $\begin{array}{c}0,093 \\
\pm \\
0,013\end{array}$ & $\begin{array}{c}0,031 \\
\pm \\
0,004\end{array}$ & $\begin{array}{c}0,061 \\
\pm \\
0,010\end{array}$ & $\begin{array}{c}0,132 \\
\pm \\
0,020\end{array}$ & $\begin{array}{c}0,124 \\
\pm \\
0,013\end{array}$ & $\begin{array}{c}0,135 \\
\pm \\
0,048\end{array}$ & $\begin{array}{c}0,173 \\
\pm \\
0,042\end{array}$ \\
\hline $\begin{array}{c}\text { Grupo } 2 \\
\text { (Gln) }\end{array}$ & $\begin{array}{c}0,244 \\
\pm \\
0,045\end{array}$ & $\begin{array}{c}0,227 \\
\pm \\
0,024\end{array}$ & $\begin{array}{c}0,055 \\
\pm \\
0,005\end{array}$ & $\begin{array}{c}0,051 \\
\pm \\
0,012\end{array}$ & $\begin{array}{c}0,020 \\
\pm \\
0,007\end{array}$ & $\begin{array}{c}0,039 \\
\pm \\
0,011\end{array}$ & $\begin{array}{c}0,080 \\
\pm \\
0,010\end{array}$ & $\begin{array}{c}0,100 \\
\pm \\
0,010\end{array}$ & $\begin{array}{c}0,102 \\
\pm \\
0,077\end{array}$ & $\begin{array}{c}0,164 \\
\pm \\
0,038\end{array}$ \\
\hline
\end{tabular}

Resultados expressos como Média \pm E.P.M. (n=6, cada subgrupo)

FIGURA 4 - Representação gráfica das concentrações de uréia na urina dos animais dos grupos 1 e 2 nos dias 1 e 2 do estudo.

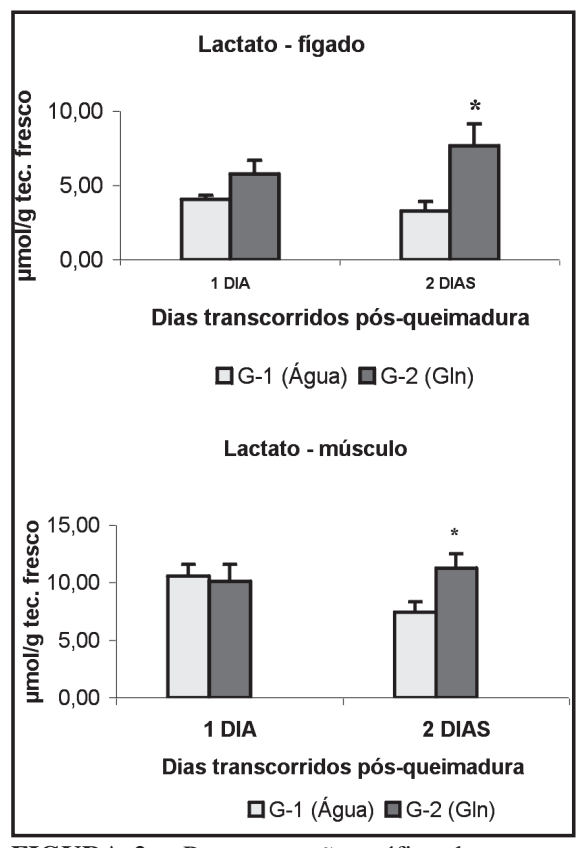

FIGURA 2 - Representação gráfica da concentração de lactato no fígado e músculo dos animais dos grupos 1 e 2 nos dias 1 e 2 do estudo.

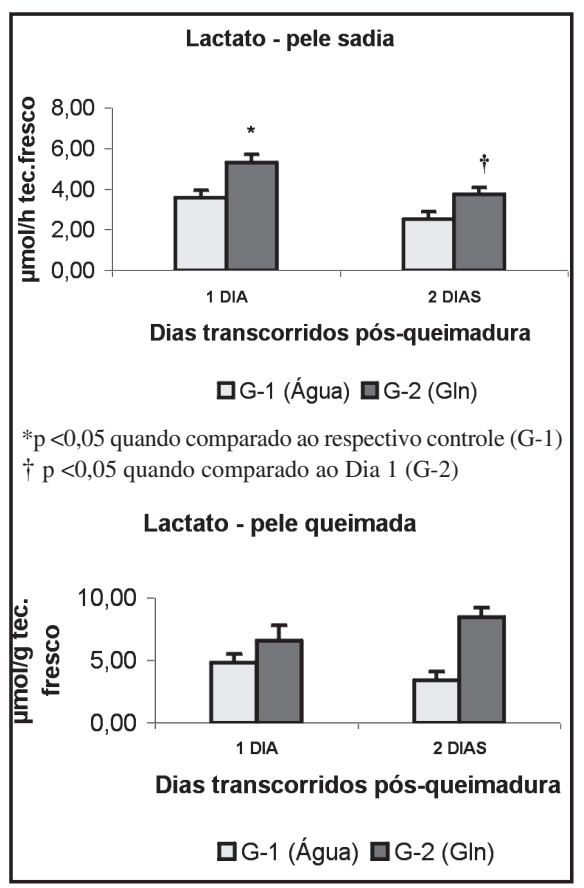

FIGURA 3 - Representação gráfica da concentração de lactato na pele sadia e queimada dos animais dos grupos 1 e 2 nos dias 1 e 2 do estudo.

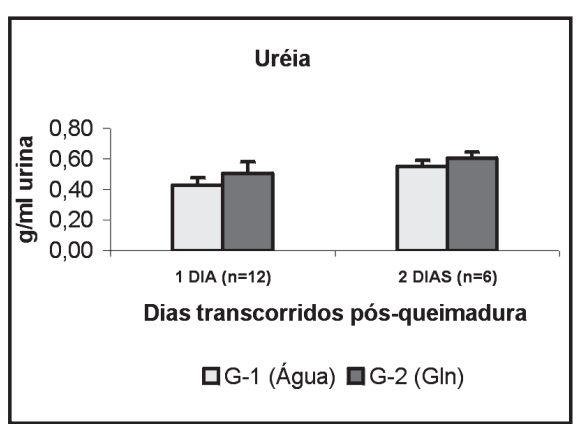


substratos da periferia incluindo o lactato, piruvato e os aminoácidos gliconeogênicos (proteólise), tais como a alanina e a glutamina. Como a lipólise também aumenta nas queimaduras é possível que o principal combustível oxidado seja o lipídio e não o carboidrato, a despeito da elevação marcante da síntese de glicose no fígado. O metabolismo protéico exibe, após queimaduras, um marcante aumento na quebra de proteínas. O mecanismo específico é desconhecido e está possivelmente relacionado aos hormônios do estresse, as citocinas, dentre as quais a interleucina 1 , e aos oxidantes.

O elevado consumo de oxigênio após uma grande queimadura é decorrente de uma maior utilização de energia produzida a partir de uma maior oxidação de substratos. Entretanto, torna-se importante determinar se o aumento prolongado da taxa metabólica causa adaptações na habilidade dos tecidos lesados em utilizar ou prover substratos e energia. A determinação das concentrações in vivo das concentrações de metabólitos (glicose, piruvato, lactato, corpos cetônicos, ATP) e da uréia urinaria são bons indicadores do comportamento das vias metabólicas de produção de energia, e da quebra de proteína, em reposta a grandes queimaduras, tendo sido por este motivo, alvo do presente estudo.

A redução nas concentrações in vivo de ATP no músculo no grupo tratado com Gln em relação àquelas aferidas no grupo controle nas $24 \mathrm{~h}(0,004 \pm 0,001$ $\mu \mathrm{mol} / \mathrm{g}$ versus $0,010 \pm 0,002 \mu \mathrm{mol} / \mathrm{g}-\mathrm{p}<$ $0,05)$ e $48 \mathrm{~h}(0,002 \pm 0,001 \mu \mathrm{mol} / \mathrm{g}$ versus $0,009 \pm 0,002 \mu \mathrm{mol} / \mathrm{g}-\mathrm{p}<0,05)$ pós-trauma térmico, sugere uma predominância da glicólise anaeróbica para manutenção da produção de energia. A oferta de Gln exógena propiciou maior disponibilidade de glutamato levando à ativação da lançadeira malato-aspartato com consequente formação de um ambiente pró-glicolítico rico em NAD ${ }^{+}$(Figura 2). Sabe-se que, com o processo glicolítico anaeróbico, ocorre menor produção de ATP do que ao ser utilizada a glicólise aeróbica.

Tang e col. (1994) encontraram um aumento significativo na glicemia em ratos queimados, 3 horas após a injúria, persistindo elevada até 48 horas, caindo lentamente após este período. ${ }^{13}$ Não foram observadas, no presente estudo, altera- ções na glicemia entre os grupos de animais analisados (Tabela 2). O aumento significante na concentração de glicose na pele sadia dos ratos tratados com Gln, no dia 2 em comparação com o grupo controle $(1,129 \pm 0,105 \mu \mathrm{mol} / \mathrm{g}$ versus 0,644 $\pm 0,167 \mu \mathrm{mol} / \mathrm{g}-\mathrm{p}<0,05)$ sinaliza um aumento na captação de glicose por este tecido (Figura 3). Na pele queimada houve aumento significante $(1,916 \pm 0,201 \mu \mathrm{mol} /$ $\mathrm{g}$ versus $0,914 \pm 0,218 \mu \mathrm{mol} / \mathrm{g}-\mathrm{p}<0,05)$ na concentração de glicose no grupo tratado com Gln em relação ao grupo controle no primeiro dia pós-trauma térmico. Por outro lado houve uma diminuição $(0,665 \pm 0,282$ $\mu \mathrm{mol} / \mathrm{g}$ versus $1,916 \pm 0,201 \mu \mathrm{mol} / \mathrm{g}-$ $\mathrm{p}<0,05)$ no segundo dia pós-trauma quando comparadas às concentrações determinadas no primeiro dia no mesmo grupo (G-2) (Tabela 2). A oferta de Gln induziu, portanto, possivelmente aumento na captação de glicose tanto por pele sadia quando na pele queimada, sendo tal fato mais intenso no dia 1 pós-trauma térmico.

O piruvato desempenha um papel central importante no metabolismo dos carboidratos e lipídios. Tem parceria íntima com o lactato e a glicose. É o produto final da via glicolítica, onde acontece uma série de reações, convertendo $1 \mathrm{~mol}$ de glicose em 2 moles de piruvato, produzindo-se 2 moles de ATP.

No presente estudo não foram encontradas alterações significantes nas concentrações de piruvato no sangue em nenhum dos grupos estudados. A queda nas concentrações de piruvato no fígado $(0,054 \pm 0,010 \mu \mathrm{mol} / \mathrm{g}$ versus $0,173 \pm 0,016$ $\mu \mathrm{mol} / \mathrm{g} \mathrm{p}<0,05)$ no primeiro dia pós-trauma no grupo que utilizou Gln em comparação com o controle $(\mathrm{G}-1)$ pode significar aumento do consumo deste metabólito, possivelmente como substrato para gliconeogênese hepática (Figura 3). A queda nas concentrações de piruvato no músculo $(0,008 \pm 0,003 \mu \mathrm{mol} / \mathrm{g}$ versus $0,04 \pm 0,006$ $\mu \mathrm{mol} / \mathrm{g}-\mathrm{p}<0,05)$ no dia 1 pós-queimadura, no grupo tratado com Gln, em relação ao controle, sugere um maior consumo de piruvato, para produção de energia no ciclo do ácido tricarboxílico (Figura 3). O aumento nas concentrações de piruvato no dia 2 pós-trauma dos ratos recipientes de Gln, em relação ao dia 1 , na pele sadia $(0,022 \pm 0,004 \mu \mathrm{mol} / \mathrm{g}$ versus $0,006 \pm 0,002$ $\mu \mathrm{mol} / \mathrm{g}-\mathrm{p}<0,05)$ pode sugerir maior captação deste substrato pela pele sadia
(Figura 4). A queda nas concentrações de piruvato do dia 1 para dia $2(0,017 \pm 0,006$ $\mu \mathrm{mol} / \mathrm{g}$ versus $0,097 \pm 0,067 \mu \mathrm{mol} / \mathrm{g}-$ $\mathrm{p}<0,05$ ) na pele queimada (grupo controle) pode significar menor captação ou aumento do consumo deste metabólito no ciclo do ácido tricarboxílico (Figura 4). Um maior consumo de piruvato por parte do tecido cutâneo lesado no D2 poderia ser explicado por aumento do gasto energético decorrente do processo de reparação pós-trauma térmico.

O lactato é continuamente formado por muitos tecidos sendo que, em queimaduras, o local primário da produção aumentada de lactato é a pele acometida de lesão térmica. O mecanismo para o aumento na síntese de lactato seria a hipoperfusão tecidual ou a disfunção mitocondrial. ${ }^{12}$ Tang e col. (1994) encontraram uma elevação significativa na lactacemia em ratos queimados, com pico após 6 horas, declinando lentamente até o terceiro dia pós-trauma. ${ }^{13}$

Carter e col. (1993), não encontraram alterações nas concentrações hepáticas de glicose e lactato num período de 48 horas após queimadura de $20 \%$ e espessura total da pele, em ratos, sugerindo que as alterações metabólicas sistêmicas encontradas no trauma térmico são predominantemente associadas com mudanças metabólicas nos tecidos periféricos e não necessariamente com o fígado. ${ }^{14}$

No presente estudo não foram observadas alterações significantes na lactacemia entre os grupos estudados, apesar da lactacemia encontrar-se elevada em ambos os grupos de animais em decorrência à lesão térmica a que foram submetidos. $\mathrm{O}$ aumento na concentração de lactato no fígado $(7,680 \pm 1,490 \mu \mathrm{mol} / \mathrm{g}$ versus 3,272 $\pm 0,675 \mu \mathrm{mol} / \mathrm{mg}-\mathrm{p}<0,01)$ no dia 2 , nos ratos do grupo G-2, em relação ao controle (G-1), pode ser decorrente do aumento da captação deste metabólito pelo tecido hepático, provavelmente devido a uma ativação do Ciclo de Cori no período pósqueimadura (Figura 4). Este fato é corroborado pelo aumento nas concentrações musculares de lactato $(11,290 \pm 1,250$ $\mu \mathrm{mol} / \mathrm{g}$ versus $7,430 \pm 0,930 \mu \mathrm{mol} / \mathrm{g}-$ $\mathrm{p}<0,05$ ) no grupo G-2, em comparação às concentrações aferidas no G-1, também no segundo dia pós-queimadura devendo-se, provavelmente, à ativação da glicólise 
anaeróbica (Figura 4). Na pele sadia observou-se aumento significante nas concentrações de lactato $(5,300 \pm 0,420 \mu \mathrm{mol} / \mathrm{g}$ versus $3,570 \pm 0,389 \mu \mathrm{mol} / \mathrm{g}-\mathrm{p}<0,05)$ dos ratos tratados com Gln (G-2), no dia 1, em comparação às concentrações aferidas no G-1, no mesmo período. Houve ainda, em pele sã, redução significante das concentrações de lactato $(5,300 \pm 0,420 \mu \mathrm{mol} / \mathrm{mg}$ versus $3,570 \pm 0,380 \mu \mathrm{mol} / \mathrm{mg} \mathrm{p}<0,05)$ no dia 2 em relação às concentrações aferidas no dia 1 , nos animais tratados com Gln. Estas alterações periféricas podem ser devidas ao aumento da glicólise anaeróbica que declina com o passar do tempo pósqueimadura. $\mathrm{O}$ aumento da glicólise anaeróbica, em animais recipientes de Gln, pode dever-se a uma captação deste aminoácido e sua transformação em glutamato, com ativação do ciclo malato-aspartato, levando ao favorecimento da oferta de $\mathrm{NAD}^{+}$ no citosol, com aumento da atividade glicolítica anaeróbica. ${ }^{15}$ Não se observaram alterações significantes na concentração de lactato na pele queimada em nenhum dos grupos estudados. É possível que a magnitude da lesão térmica acarrete falência na energética tecidual e eventuais diferenças induzidas pela oferta exógena de glutamina possam não ter sido passíveis de observação.

Os corpos cetônicos são importantes fontes alternativas de energia. Produzidos no fígado a partir da acetil-CoA, reação mediada pela enzima hidroximetilglutarilredutase, os corpos cetônicos são importantes combustíveis alternativos, na ausência da glicose.

As concentrações de corpos cetônicos estão freqüentemente aumentadas no jejum. A ausência da hipercetonemia do jejum poderia ser explicada pela menor produção de corpos cetônicos pelo fígado, decorrente da hiperinsulinemia que ocorre com o trauma térmico, tendo como conseqüência uma menor disponibilidade destes compostos na corrente sanguínea, menor captação e queda na sua utilização pelos tecidos periféricos. No presente estudo o trauma térmico manteve as concentrações sanguíneas de corpos cetônicos baixas tanto no grupo de estudo quando no grupo controle, mesmo após o jejum de $48 \mathrm{~h}$, caracterizando a falha de desenvolvimento da hipercetonemia adaptativa ao jejum (Tabela 4).
Tischler e col. (1983), relataram quantidades aumentadas de uréia urinária em ratos traumatizados, com pico em torno do terceiro dia. ${ }^{16}$ A liberação pelo músculo de aminoácidos gliconeogênicos, tais como a alanina e a glutamina, encontra-se aumentada após queimadura. Os aminoácidos oriundos da proteólise muscular são deaminados com aumento da ureagênese. ${ }^{17} \mathrm{~A}$ oferta exógena de Gln não induziu alteração na ureagênese já que, no presente estudo, não se verificaram alterações pós-queimadura significantes, na uréia urinária, em nenhum dos grupos estudados.

A oferta exógena de L-glutamina pela via enteral, através de gavagem, promoveu importantes alterações no metabolismo intermediário no período até 48 horas queimadura, no presente modelo. Gln induziu aumento na capacidade de síntese de glicose pela via anaeróbica no músculo e pele saudável, por provável ativação do ciclo malato-aspartato. Houve também maior disponibilidade de lactato para o fígado para fins de gliconeogênese. Tais alterações, dentre outras observadas no presente estudo, podem vir a ser adaptativas e pró-hospedeiro. A ativação do ciclo de Cori com utilização de lactato como precursor de glicose no fígado, por exemplo, poderia caracterizar uma ação poupadora de proteína muscular no período pós-trauma térmico. Entretanto, mais estudos metabólicos cinéticos in vivo utilizando L-glutamina marcada em modelos experimentais semelhantes são necessários para ajudar elucidar os efeitos da Gln sobre o metabolismo intermediário na vigência de queimadura.

\section{Conclusões}

1. A oferta de L-glutamina, por gavagem, provoca queda nas concentrações in vivo de piruvato e ATP no músculo, $24 \mathrm{~h}$ após a indução de trauma térmico, sugerindo utilização de piruvato para glicólise preponderantemente anaeróbica.

2. A administração de L-glutamina, por gavagem, provoca aumento nas concentrações in vivo de lactato no fígado e músculo, 48 pós-trauma térmico, sugerindo ativação do ciclo de Cori. O aumento nas concentrações de lactato tecidual pode vir a ser decorrente da oferta exógena de Gln, que transformada em glutamato, leva à ativação do ciclo malato-aspartato com conseqüente favorecimento da glicólise anaeróbica.

3. A oferta de Gln induz a possível aumento na captação de glicose tanto por pele sadia quando na pele queimada, sendo tal fato mais intenso no dia 1 pós-trauma térmico.

4. O trauma térmico neste modelo mantém as concentrações sanguíneas de corpos cetônicos baixas tanto no grupo de estudo quando no grupo controle, mesmo após o jejum de 48 h, caracterizando falha de desenvolvimento da hipercetonemia adaptativa ao jejum

5. A oferta exógena de Gln não induz alteração na ureagênese já que não se verificaram alterações pós-queimadura significantes na uréia urinária de 24 h, quando comparados os grupos: recipiente de Gln e controle.

\section{Referências}

1. Wolfe RR. Relation of metabolic studies to clinical nutrition-the example of burn injury. Am J Clin Nutr 1996;64:800-8.

2. Voet D, Voet JG, Pratt CW.Origem da vida. In:_______.Fundamentos de Bioquímica. 1ed. Porto Alegre: Artemed;2000. p 4-21.

3. Lustosa AMP. Efeito da glutamina na permeabilidade intestinal, evolução clinica e toxicidade renal em crianças com diarréia persistente e desnutrição. [Dissertação Mestrado]. Universidade Federal do Ceará - Faculdade de Medicina; 2001.

4. Walker HL, Mason AD. A standard animal burn. J. Trauma 1968;8:1049-51.

5. Moncrief JA. In: Artz CP, Moncrief JA, Pruitt BA. (Ed). Burns: A team approach. Philadelphia: W.B.Saunders Company, 1979.

6. Lohmann R, Souba WW, Zakrzewski K, Bode BP. Stimulation of rat hepatic amino acid transport by burn injury. Metabolism 1998;47:608-16.

7. Pawlik TM, Lohmann, R, Souba WW, Bode BP. Hepatic glutamine transporter activation in burn injury: role of amino acids and phosphatidylinositol-3-kinase. Am J Physiol Gastrointest Liver Physiol 2000;278:G532-41.

8. Hohorst HJ. D-Glucose-6-phosphate and Dfructose-6-phosphate. Determination with glucose-6-phosphate dehydrogenase and phosphoglucose isomerase. In: Bergmeyer HU. Methods of Enzymatic Analysis. Verlag Chemie. London: Weinheim/Academic Press;1963. p.134-8. 
9. Williamson DH, Mellanby J, Krebs HA. Enzymic determinations of the D-(-) Bhydroxybutyric acid and acetoacetic acid in blood. Biochem J 1962;82:90-6.

10. Slein MW. Determination with hexokinase and glucose-6-phosphate dehydrogenase. In: Bergmeyer HU. Methods of Enzymatic Analysis. Verlag Chemie, London: Weinheim/Academic Press 1963. p.117-23.

11. Jordão AAJR, Lourenço EJ, Vitali LH, Dutra de Oliveira JE, Marchini JS. Excreção urinária de nitrogênio total, creatinina e uréia na avaliação nutricional. Cad Nutr 1992;5:1.

12. Demling RH, Seigne P. Metabolic management of patients with severe burns. World J Surg 2000;24:673-80.

13. Tang YW, Fang RH, Yu YM, Burke JF. Enhanced amino acid uptake in both skeletal muscle and liver by burn plasma in rats. Burns 1994;20:508-13.

14. Carter EA, Khalid MA, Burke JF, Tompkins RG. Absence of change in hepatic lactate metabolism after burn injury. Burns 1993; (6) $475-8$.
15. Barron JT, Gu L, Parrilo JE. Malateaspartate shuttle, cytoplasmic NADH redox potential, and energetics in vascular smooth muscle. J Mol Cell Cardiol 1998; 30:1571-9.

16. Tischler M, Fagan JM. Response to trauma of protein, aminoacid and carbohydrate metabolism in injuried rat skeletal muscles. Metabolism 1983;32:853-68.

17. Cynober L. Amino acid metabolism in thermal burns. J Parenter Enteral Nutr 1989; 13:196-205.

Barbosa RCC, Guimarães SB, Vasconcelos PRC, Chaves CR, Vasconcelos PRL. Metabolic effects of glutamine in rats subjected to scald burn. Acta Cir Bras [serial online] 2003 Nov-Dec;18(6). Available from URL: http://www.scielo.br/acb.

ABSTRACT - Purpose: Investigate the metabolic effects of L- glutamine (Gln) in rats subjected to scald burn. Methods: Twenty-four anesthetized male Wistar rats were submitted to scald burn of dorsal skin secondary to exposure to hot water $\left(100^{\circ} \mathrm{C}\right)$ for $10 \mathrm{sec}(30 \%$ of body surface). Eighteen and $42 \mathrm{~h}$ later rats were randomized to receive (by gavage) $2 \mathrm{ml}$ of water (G-1) or equal volume ( $0,5 \mathrm{~g} / \mathrm{Kg}$ weight/day) of Gln solution (G-2). Tissue and blood samples were collected at the end of $24 \mathrm{~h}$ (D1) and 48h (D2) post burn for enzymatic analysis. Results: Glucose concentrations were significantly increased in healthy skin in G-2/D2 and in burned skin in G-2/D1. Lactate concentrations were significantly increased in G-2/D1 in muscle $(11,29 \pm 1,25 \mu \mathrm{mol} / \mathrm{g}$ versus 7,43 $\pm 0,93 \mu \mathrm{mol} / \mathrm{g}$ $\mathrm{p}<0.05)$ and in $\mathrm{G}-2 / \mathrm{D} 2$ subgroups in liver $(7,68 \pm 1,49 \mu \mathrm{mol} / \mathrm{g}$ versus $3,27 \pm 0,67 \mu \mathrm{mol} / \mathrm{g}-\mathrm{p}<0.01)$ and healthy skin $(5,30$ $\pm 0,42 \mu \mathrm{mol} / \mathrm{g}$ versus $3,57 \pm 0,38 \mu \mathrm{mol} / \mathrm{g}-\mathrm{p}<0.05)$. Pyruvate concentrations were significantly decreased in G-2/D1 subgroups in muscle and liver and increased in healthy skin in G-2/D2 subgroups. ATP concentrations were significantly decreased in muscle in G-2/D1 and G-2/D2. Conclusions: The offer of Gln signals for preponderance of pyruvate use for anaerobic glycolysis in muscle. Increase in tissue lactate concentrations may be due to exogenous offer of Gln, that once transformed in glutamate leads to the activation of malate-aspartate shuttle with consequent enhancing of anaerobic glycolysis. Offer of Gln induces possible increase in glucose uptake both in healthy and burned skin. There was a development flaw of the fast adapting hyperketonemia in both groups. Exogenous offer of Gln seems not to induce alteration in urea genesis.

KEY WORDS - Burns. Metabolism. Glutamine. Rats.

Correspondência:

Conflito de interesse: nenhum

Paulo Roberto Leitão de Vasconcelos

Departamento de Cirurgia

Rua Prof. Costa Mendes, $1608 / 3^{\circ}$ andar

60430-140 Fortaleza - Ceará

Tel.: (85)288-8063

Fax: (85)288-8064

mcirur@npd.ufc.br

Fonte de financiamento: nenhuma

Data do recebimento: 11/09/2003

Data da revisão: 01/10/2003

Data da aprovação: 21/10/2003 